\title{
Phenotype characteristics of gastric epithelial mucus in patients with different gastric diseases: from superficial gastritis to gastric cancer
}

\author{
Nannan Dong ${ }^{1,2,3}$, Rui Guo ${ }^{1,2,3}$, Yuehua Gong ${ }^{\text {Corresp., 1, 2, } 3}$, Yuan Yuan ${ }^{\text {Corresp. 1, 2, } 3}$ \\ 1 the First Hospital of China Medical University, Key Laboratory of GI Cancer Etiology and Prevention in Liaoning Province, Shenyang, LiaoNing, China \\ 2 the First Hospital of China Medical University, Tumor Etiology and Screening Department of Cancer Institute and General Surgery, Shenyang, LiaoNing, \\ China \\ 3 the First Hospital of China Medical University, Key Laboratory of Cancer Etiology and Prevention in Liaoning Education Department, Shenyang, LiaoNing, \\ China \\ Corresponding Authors: Yuehua Gong, Yuan Yuan \\ Email address: yhgong@cmu.edu.cn, yuanyuan@cmu.edu.cn
}

Background $\square$ Gastric gland mucin is important for maintaining the basic function of the gastric mucosa, protecting it from foreign substances and reducing the occurrence of gastric diseases. Exploring the phenotype of gastric gland mucus changes during the progression of gastric disease is of great clinical significance.Methods $\square$ A total of 483 patients with different gastric diseases were collected in this study, including 82 superficial gastritis (SG), 81 atrophic gastritis (AG), 168 dysplasia (GD), and 152 gastric cancer (GC). Mucin staining was performed using HID-ABpH2.5-PAS method and was further grouped according to the mucin coloration.Results: The phenotypic characteristics of mucin during disease progression were divided into neutral, acidic, and mucus-free types. Furthermore, acidic mucus can be divided into type I, type II, and type III. The SG group was dominated by neutral mucus (100\%), and the AG was dominated by acid mucus ( $81.48 \%)$, which gradually increased with the severity of atrophy $(P<0.05)$. The GD and GC groups were dominated by mucus-free $(43.45 \%, 78.29 \%)$, and as the degree of GD worsened, neutral and acidic mucus gradually decreased and mucus-free increased $(P<0.001)$. From the $S G$, AG, GD, and GC progression, neutral and acidic mucus gradually decreased, and mucusfree gradually increased. Acidic mucin revealed that type III (red-brown black) mucin was predominant in $A G, G D$, and $G C$, and increased with the degree of $A G, G D$, as well as the biological behavior of GC. In the lesion adjacent to high-grade GD or GC, type III acid mucin is predominant.Conclusion $\square$ There were three mucin phenotypes in the process of gastric diseases. With the disease progression, the trend of phenotypic change was that neutral and acidic mucus gradually decreased and mucus-free increased. The appearance of type III mucin suggested a relatively serious phase of gastric diseases and may be a more suitable candidate for follow-up monitoring of patients with GC risk. 
1 Phenotype characteristics of gastric epithelial mucus in patients with different

\section{gastric diseases: from superficial gastritis to gastric cancer} Nannan Dong, Rui Guo, Yuan Yuan*, Yuehua Gong*

${ }^{1}$ Tumor Etiology and Screening Department of Cancer Institute and General Surgery, the First Hospital of China Medical University, Shenyang 110001, China

${ }^{2}$ Key Laboratory of Cancer Etiology and Prevention in Liaoning Education Department, the First Hospital of China Medical University, Shenyang 110001, China

${ }^{3}$ Key Laboratory of GI Cancer Etiology and Prevention in Liaoning Province, the First Hospital of China Medical University, Shenyang 110001, China

\section{*Co-Correspondence should be addressed to}

Dr. Gong Yuehua, Tumor Etiology and Screening Department of Cancer Institute and General Surgery, The First Hospital of China Medical University, No.155 NanjingBei Street, Heping District, Shenyang, Liaoning Province, P.R. China 110001, Telephone: +86-024-83282153; fax: +86-024-83282383. Email: yhgong@cmu.edu.cn

Dr. Yuan Yuan, Tumor Etiology and Screening Department of Cancer Institute and General Surgery, The First Hospital of China Medical University, No.155 NanjingBei Street, Heping District, Shenyang, Liaoning Province, P.R. China 110001, Telephone:+86-02483282153;fax:+86-024-83282292.Email: yuanyuan@.cmu.edu.cn 
Background: Gastric gland mucin is important for maintaining the basic function of the gastric mucosa, protecting it from foreign substances and reducing the occurrence of gastric diseases. Exploring the phenotype of gastric gland mucus changes during the progression of gastric disease is of great clinical significance.

Methods : A total of 483 patients with different gastric diseases were collected in this study, including 82 superficial gastritis (SG), 81 atrophic gastritis (AG), 168 dysplasia (GD), and 152 gastric cancer (GC). Mucin staining was performed using HID-ABpH2.5-PAS method and was further grouped according to the mucin coloration.

Results: The phenotypic characteristics of mucin during disease progression were divided into neutral, acidic, and mucus-free types. Furthermore, acidic mucus can be divided into type I, type II, and type III. The SG group was dominated by neutral mucus (100\%), and the AG was dominated by acid mucus $(81.48 \%)$, which gradually increased with the severity of atrophy $(P<0.05)$. The GD and GC groups were dominated by mucus-free (43.45\%, 78.29\%), and as the degree of GD worsened, neutral and acidic mucus gradually decreased and mucus-free increased $(P<0.001)$. From the SG, AG, GD, and GC progression, neutral and acidic mucus gradually decreased, and mucus-free gradually increased. Acidic mucin revealed that type III (red-brown black) mucin was predominant in $\mathrm{AG}, \mathrm{GD}$, and $\mathrm{GC}$, and increased with the degree of $\mathrm{AG}, \mathrm{GD}$, as well as the biological behavior of GC. In the lesion adjacent to high-grade GD or GC, type III acid mucin is predominant.

Conclusion: There were three mucin phenotypes in the process of gastric diseases. With the disease progression, the trend of phenotypic change was that neutral and acidic mucus gradually decreased and mucus-free increased. The appearance of type III mucin suggested a relatively serious phase of gastric diseases and may be a more suitable candidate for follow-up monitoring of patients with GC risk. 
57 Key words: gastric mucus, gastric disease, HID-ABpH2.5-PAS

58

59

Mucins, a family of large and heavily glycosylated proteins, represent the main component of this hydrophilic gel-like mixture, and act as key molecules in the maintenance of gastrointestinal homeostasis ${ }^{[1,2]}$ and protect the gastric mucosa from the damage from external environment ${ }^{[3,4]}$. These mucins are divided into two subtypes: surface mucin and gland mucin ${ }^{[5,6]}$. The former is secreted from the surface cells of the gastric mucosa, while the latter is located in the lower layer of the gastric mucosa from the gland mucous cells. The stomach wall covered with surface mucus will not be directly exposed to the gastric juice environment with strong acid and proteases. Under normal circumstances, surface mucin can reduce gastric mucosal irritation caused by food friction, resist pepsin damage to gastric mucosa and reduce $\mathrm{H}^{+}$penetration to maintain the mucusbicarbonate barrier function ${ }^{[7]}$. Surface mucus is also important in drugs absorption process, namely for those that are gastric $\mathrm{pH}-$ sensitive $^{[8]}$. Gastric gland mucins are derived from pyloric glands, mucus neck cells, and fundus gland cells ${ }^{[9]}$. These mucus are important for maintaining the basic function of the gastric mucosa, protecting against foreign substances, and reducing the occurrence of gastric diseases ${ }^{[10]}$.

The occurrence of GC is due to the chronic inflammation of the gastric mucosa under the longterm effect of pathogenic factors, which can further cause AG, IM(intestinal metaplasia), GD and $\mathrm{GC}^{[11]}$ following the Correa's Model. Exploring the changes of mucus phenotype during the above pathological process is of great significance to reveal the occurrence and development of gastric diseases. It has been reported that expression and glycosylation of mucins are associated with gastric carcinogenesis including invasion, proliferation and regulation of tumor cells ${ }^{[12-15]}$. Several studies have suggested that the type of mucin expressed in early and advanced gastric carcinomas is of clinical significance, alluding to biologic differences in precursor lesions and/or pathways of malignant transformation ${ }^{[16-19]}$. Accumulating evidence has indicated that different mucin 
83 phenotypes of GC have distinct clinical characteristics and exhibit specific genetic and epigenetic

84 changes ${ }^{[20]}$. Thus, mucin phenotype classification is useful to understand GC pathogenesis.

85 However, it is not exactly clear how the phenotype of gastric gland mucus changes during the 86 progression of gastric disease.

87 In this study, we used AB-PAS mucus staining method to explore the changes of mucus 88 phenotype during the occurrence and development of gastric diseases, which would provide clues 89 and help to reveal the occurrence and development of gastric diseases ${ }^{[21]}$.

90 Material and Methods :

91 1) The subjects

92 A total of 483 patients with different gastric diseases were included in this study, among them

93283 patients underwent gastroscopic biopsy and 118 patients underwent endoscopic submucosal 94 dissection (ESD) surgery at the Endoscopy Center of the First Affiliated Hospital of China Medical 95 University from July 2014 to March 2018. In addition, 82 cases from the on-site GC screening 96 project in Zhuanghe county, Liaoning province from January 2014 to December 2014. They were diagnosed as 82 SG, 81 AG, 168 GD, 152 GC, including 299 males and 184 females, with an average age of 60.25 years old. The $H$. pylori infection was detected using ASSURE $H p$ rapid test (MP Diagnostics ${ }^{\mathrm{TM}}$ ). The histopathological diagnostic criteria of the subjects referred to the updated Sydney classification system. This study was approved by the Ethics Committee of the First Affiliated Hospital of China Medical University, and the subjects signed informed consents ([2013]135 and 2016[161]).

\section{2)HID-ABpH2.5-PAS mucin histochemical staining}

The specific steps of HID-ABpH2.5 PAS mucin histochemical staining followed the instructions of the kit ( kit was purchased from Beijing Regen Biotechnology Co., Ltd.). In short, after dewaxing to water, the slides were placed in the solution (A1:A2=50:3), away from light for $18-24$ hours at $24^{\circ} \mathrm{C}-28^{\circ} \mathrm{C}$. After that, stained with Alcian blue for 20 minutes, and next incubated with periodic acid for 30 seconds, and then schiff reagent was added for 2 minutes, and at last the 
110 material is blue, mixed mucus material is blue-purple or purple-blue, and the nucleus is brown.

\section{3 ) The phenotypes of different acid mucus}

112 In acid mucus, sialic acid mucus is blue, and sulfuric acid mucus is brownish black. Further,

113 according to the different coloration of acid mucin, the gastric mucosa is divided into type I (blue

114 or brown-black), type II (red-blue) and type III (red-brown black). If there is more than one mucus

115 phenotype in the lesion, the most important mucus phenotype is classified.

116 4) Statistical analysis

117 SPSS 16.0 software was used for statistical analysis. $P<0.05$ was statistically significant. The

118 descriptive statistics of continuous variables are mean, standard deviation, minimum and

119 maximum, while categorical variables are count and percentage. Chi-square test or Fisher's exact

120 test were used to determine the statistical association between categorical variables, and test was

121 used to determine the statistical association between continuous variables.

122

123 Results :

124 1) The baseline characteristics of the subjects

125 A total of $82 \mathrm{SG}, 81 \mathrm{AG}, 168 \mathrm{GD}$, and $152 \mathrm{GC}$ were included in this study, the average age 126 and gender composition of each group were shown in Table 1, of which the SG group was younger 127 compared with other groups, with statistical significance $(P=0.000)$. In terms of male and female 128 composition, the difference between the groups was not statistically significant $(P=0.067)$.

\section{2) Mucous phenotype characteristics of different gastric diseases}

130 We compared the mucin staining of mucosal tissues in different gastric diseases, as shown in 131 Table 2 and Figure 1. Of the total 483 patients, 135 (27.89\%), $154(31.82 \%)$, and $195(40.29 \%)$

132 were neutral, acidic mucus, and mucus-free respectively. Among them, 100\% of the SG group was 133 neutral mucus. The patients with AG were mainly acid mucus, accounting for $81.48 \%$, followed

134 by neutral mucus accounting for $14.81 \%$, and the difference between the groups was statistically 135 significant $(P=0.000)$. The patients with GD were mainly without mucus $(43.45 \%)$, followed by 136 acidic mucus $(35.12 \%)$ and neutral mucus $(21.43 \%)$, the difference between the groups was 
137 statistically significant $(P=0.000)$; patients with GC were similar to patients with GD, and mainly 138 stained without mucus (78.29\%), the rest were neutral mucus (2.63\%) and acid mucus (19.08\%).

139 The difference between the groups was statistically significant $(P=0.000)$.

140 In the comparison between different disease groups, the expression of neutral mucus 141 gradually decreased from SG, AG to GD and GC, with a positivity of $100 \%, 14.81 \%, 21.43 \%$ and $1422.63 \%$, respectively, and the difference between the groups was statistically significant $(P=0.000)$.

143 The expression of acidic mucus gradually decreased (81.48\%, 35.12\% and 19.08\%) respectively,

144 the difference between the groups was statistically significant $(P=0.000)$. Those mucus-free 145 expression gradually increased $(3.71 \%, 43.45 \%$ and $78.29 \%)$ respectively, the difference between 146 the groups was statistically significant $(P=0.000)$. It can be seen that $\mathrm{SG}$ was mainly composed of 147 neutral mucus, AG was mainly expressed by acidic mucus, and GD and GC tissues were mainly 148 expressed by mucus-free. With the progress of gastric disease, neutral and acidic mucus expression 149 gradually decreased, and those without mucus expression gradually increased.

3 ) Subgroup analysis of mucous phenotype characteristics of different gastric diseases

151 Further, we analyzed mucin staining in different subgroups of AG, GD, and GC. The results

152 were shown in Table 3. In AG, there was no statistically significant difference in the percentage 153 of neutral mucus and acid mucus in the antrum, corpus and angle of stomach $(P=0.874)$. In mild 154 atrophy, neutral and acid mucus accounted for 33.33 and $53.33 \%$, respectively. The acid mucus of 155 moderate and severe AG were $85.11 \%$ and $94.74 \%$, respectively, higher than that of mild AG, and 156 the difference between the groups was statistically significant $(P=0.02)$. In the low-grade of GD, 157 neutral, acidic mucus, mucus-free were 34.38\%, 50.00\%, 15.62\%, and in the high-grade were $1584.17 \%, 15.28 \%, 80.56 \%$, the difference between the groups was statistically significant $(P=0.000)$.

159 In GC, mucus-free was the highest in intestinal-type, followed by diffuse-type, unclassifiable type 160 and mixed-type, but the difference was not statistically significant $(P=0.5)$. It can be seen that in 161 AG, acid mucus gradually increases with the severity of gastric disease, in GD, as the degree 162 increasing, neutral and acid mucus gradually decreased, and no mucus increased. Among various 163 histological types of GC, those with no mucus were the highest. 
164

165

166

167

168

169

170

171

172

173

174

175

176

177

178

179

180

181

182

183

184

185

186

187

188

189

\section{4) The analysis of mucous phenotype characteristics of different $\boldsymbol{H}$. pylori status}

Among the AG, GD, and GC samples included in this study, there were 151 patients with $H$. pylori infection information. We compared the relationship of $H$. pylori infection status and different mucus phenotypes in the overall disease group and among the three disease groups, respectively. As shown in table 4, the results indicated that in the overall disease group, there was no difference in mucus phenotype between the $H$. pylori positive and negative groups $(P=0.724)$. However in AG subgroup, the H. pylori positive group was mainly acidic mucus type (72.97\%), and the $H$. pylori negative group was mainly neutral mucus $(100 \%)$. There was no difference in mucus phenotype between $H$. pylori positive and negative groups in GD, GC subgroups $(P=0.879,0.819)$.

\section{5) The distribution of acidic mucus phenotype in different diseases}

We further conducted a comparative analysis of acidic mucus phenotypes among different gastric diseases, including AG, GD and GC, as shown in Table 5 and Figure 2. The results showed that the acidic mucus phenotype can be further subdivided into three subtypes: type I, sialic acid or mucin sulfate; type II, neutral and acidic sialic acid mucin, occasionally, mucin sulfate, or both; type III, neutral and sulfuric acid mucus mainly, occasionally, sialic acid mucin, or both.

Among 66 cases of AG, there was 11 cases of type I (16.67\%), 10 cases of type II (15.15\%) and 45 cases of type III (68.18\%); in 59 cases of GD, there was 3 cases of type I (5.08), 12 cases of type II (20.34\%) and 44 cases of type III (74.58\%); in terms of 29 cases of GC, there were 2 cases of type I (6.9\%), 6 cases of type II (20.69\%) and 21 cases of type III (72.41\%).

Further stratified according to location and degree in AG, there was no statistically significant difference in mucin subtype $(P=0.348, P=0.505)$. In the 59 cases of GD, including 48 cases of lowgrade and 11 cases of high-grade, type III were the mainly subtype, especially with $100 \%$ in the high-grade $(P=0.000)$. There were 12 cases of intestinal-type GC and 11 cases of diffuse-type GC with mucin subtype, all of which were mainly type III, and the difference between the groups was not statistically significant $(P=0.091)$. 

GC

There were 112 cases of IM nearby the above-mentioned high-grade GD and GC. In order to further analyze the correlation between different mucus phenotypes and high-grade GD or GC. As shown in Table 6 and Figure 3, in high-grade GD, intestinal and diffuse GC, type III acid mucin is predominant, without statistically significant difference between groups $(P=0.714)$.

\section{Discussion :}

198 Current research findings suggest that mucin subtypes and clinical relevance are reported differently in the literature, but most of the data always support that different mucin subtypes are associated with the risk of intestinal gastric adenocarcinoma ${ }^{[16-19]}$. That is to say, the progression of gastric precancerous lesions to GC is a slow and gradual development process, at least targeted monitoring can be performed by mucin typing, and there is an opportunity to detect and remove tumorous lesions early. Although there are currently no clinically deterministic predictive biomarkers in clinical use, the use of mucin staining for subtype analysis can further stratify people who are already at risk ${ }^{[22]}$. In this study, we explored the changes of different mucus phenotypes during the progression of gastric disease.

Usually, the gastric epithelium, pyloric gland, and duodenal gland mainly secrete neutral mucus $^{[23]}$. The goblet cells and intestinal glands of the small and large intestine mucosa mainly secrete acidic mucus ${ }^{[24]}$. Under normal physiological conditions, the staining of gastric mucin showed red neutral mucin, but in some pathological conditions, such as IM, carcinogenesis, etc.,

211 the characteristics of gastric mucin staining can change with the specific state. Kazuhiro Yamanoi 212 et al. reported that the reduction of gastric mucus is related to the high mitotic activity of tumor 213 cells, which represents an increase in malignant potential ${ }^{[25]}$. The results of our study showed that

214 in the SG, neutral mucus was dominant, but in AG with IM, acid mucus was mainly expressed, 215 which produced by intestinal metaplasia glands. In the GD and GC, it was mainly expressed as 216 mucus-free phenotype. During the progression of gastric diseases from SG, AG, GD, and GC, the 
217 expression of neutral and acidic mucus gradually decreased, mucus-free expression gradually

218 increased. From this we can speculate that the disappearance of epithelial mucus phenotype may

219 be one of the high-risk factors for the progression of gastric disease.

220 H. pylori persistent infection of the normal gastric mucosa triggers a chronic inflammatory

221 process designated by chronic gastritis. The presence of virulent $H$. pylori strains together with

222 host immune vulnerability can lead to severe mucosal atrophy with focal loss of gland architecture

223 and disease progression ${ }^{[26]}$. In fact, the development of IM originates multiple foci where

224 superficial foveolar cells with neutral mucin expression are gradually replaced by acidic producing 225 cells with an intestinal phenotype ${ }^{[27]}$. In our study, we found in AG subgroup, the H. pylori 226 positive group was mainly acidic mucus type and the $H$. pylori negative group was mainly neutral 227 mucus. There was no difference in mucus phenotype between $H$. pylori positive and negative 228 groups in GD, GC subgroups. It was the truth that with the progress of IM, the colonization of $H$. 229 pylori gradually decreased, especially in the stage of GC, there was basically no colonization ${ }^{[28]}$, 230 so AG was dominant with acid mucus in H. pylori positive.

231 The main feature of intestinal epithelium is acid mucin staining while once IM occurs in the 232 gastric mucosa, acidic mucin can be shown in gastric epithelium. Acid mucin can be divided into 233 sialic acid or sulfated mucin, which can be used to evaluate two types using combined HID-AB 234 staining, and the latter is brown stained with ferric diamine (HID). Filipe et al. ${ }^{[29]}$ used this method 235 to distinguish mucin phenotypes: type I expressed only salivary mucin, type II expressed a hybrid 236 form of a mixture of gastric mucosa and intestinal mucin, and type III expressed neutral and 237 sulfated mucin. It is still not clear whether there is a chronological relationship between the 238 expression of these three types of mucins ${ }^{[24,30,31]}$. During the initial development of gastric 239 diseases with IM, the neutral mucin in normal mucosa gradually decreased, while salivary mucin 240 appeared and became the main type of mucin. In the serious stages of IM, sulfate mucin appeared 241 and may become the main ingredient. In this study, we detected acid mucin in AG, GD, and GC, 242 and found that type III mucin was the main type in the three types of lesions, and increased with 243 the change of the IM degree, the GD grade and the biological behavior of GC. In lesion next to 
244 high-grade GD, intestinal-type and diffuse GC, the type III acidic mucin is predominant. The 245 emergence of type III mucin is a progressive stage of IM, which can become the best candidate for 246 follow-up monitoring of patients with GC risk. In addition, based on a meta-analysis of 7 studies, 247 including patients with GIM in 2014 and no accompanying dysplasia (929 patients with type II or 248 III mucin, and 1112 patients with type I mucin), these patients ranged from 3-12.8 years of GC 249 developed during the follow-up period. Compared with individuals of type I, the risk of GC was 2503.33 times higher (95\% confidence interval, 1.96-5.64) ${ }^{[32,33]}$. Consistent with the AGA (American

251 Gastroenterological Association) guideline ${ }^{[34]}$, the recent ESGE(European Society of 252 Gastrointestinal Endoscopy) guideline also mentioned that mucin staining has prognostic value, 253 which is contrary to the 2012 ESGE guideline that opposes GIM subtype guidance for prognosis ${ }^{[35}$,

254 36]. Considering the potential prognostic value and the opportunity to generate stronger clinical and 255 epidemiological evidence, as well as the pathologist's minimum cost and effort, recommendations 256 for routine typing of mucin are advisable.

257 From a histochemical point of view, the $\mathrm{pH}$ value of normal gastric mucin is neutral, and they 258 are dyed red with periodate-Schiff (PAS). Chronic AG is histologically characterized by chronic 259 inflammation of the gastric mucosa, with decreasing number of gastric gland cells and increasing 260 intestinal metaplastic glands, therefore acid mucin replaces the original gastric mucin and gets blue 261 stained with pH 2.5 Alcian blue. Therefore, AB-PAS combined staining can distinguish normal

262 epithelium with IM. Recently, the expression profile of different mucins has been analyzed with 263 antibodies. MUC5AC, MUC6, MUC2, and CD10 have been found to be specifically expressed in 264 the gastric foveolar epithelium, pyloric gland cells, goblet cells, and brush border, respectively[17]. 265 However, this antibody staining method cannot further distinguish GI mixed type mucin into 266 salivary mucin and sulfated mucin, that is, it cannot distinguish between type II and type III. 267 Moreover, compared with antibody staining, the HID-AB-PAS method is more time-saving, 268 efficient, and cheaper and cost-effective.

269 However, the current study was limited that although we have seen the dynamic changes of 270 gastric mucus phenotype during the progression of gastric disease, whether the disappearance of 
271 mucus phenotype is "cause" or "effect", the conclusions are not consistent, and further study is 272 needed in the future such as functional assays with relevant study models (cell lines, mice...).

273 Although we found that $H$. pylori positive and negative groups have different dominant mucus 274 phenotype, however, in AG, GD, GC subgroups, the difference was not obvious. And due to the 275 limitation of the number of cases, we did not further analyze the acidic mucus subtypes between 276 H. pylori status, which has yet to be further studied.

277 In summary, this study explored the phenotype of gastric gland mucus changes during the 278 progression of gastric disease and found that the disappearance of mucus phenotype is one of the 279 high-risk factors for the progression of gastric diseases. The appearance of type III mucin is a 280 relatively serious phase of gastric diseases and may be a more suitable candidate for follow-up

281 282 283 284

291

292

293

294

295

296

297

298

299

300

301

302

303 monitoring of patients with GC risk. In China, there is currently still no effective method for clinical management and prognosis follow-up of patients with IM and dysplasia. With the continuous improvement of our understanding of mucin typing, the expansion of research samples and the increasing awareness of clinicians, we will get more evidence to support, continue to improve our clinical management of GIM and dysplasia patients, and ultimately improve the early detection rate of $\mathrm{GC}$ and reduce its mortality.

\section{References:}

1. Duarte H0, Freitas D, Gomes C, Gomes J, Magalhaes A, Reis CA: Mucin-Type 0-Glycosylation in Gastric Carcinogenesis. Biomolecules 2016, 6(3).

2. Jin C, Kenny DT, Skoog EC, Padra M, Adamczyk B, Vitizeva V, Thorel1 A, Venkatakrishnan V, Linden SK, Karlsson NG: Structural Diversity of Human Gastric Mucin Glycans. Mol Cell Proteomics 2017, 16(5) :743-758. 
304 3. Ota H, Katsuyama T: Alternating laminated array of two types of mucin in 305 the human gastric surface mucous layer. Histochem $J$ 1992, 24(2):86-92.

306 4. Amieva MR, E1-Omar EM: Host-bacterial interactions in Helicobacter

307

308

309

310

311

6. Teixeira A, David L, Reis CA, Costa J, Sobrinho-Simoes M: Expression of

312

313

314

315

7. Jin C, Kenny DT, Skoog EC, Padra M, Adamczyk B, Vitizeva V, Thorel1 A,

316

317

318

319

320

321

322

323

324

325

326

327

328

329

12. mucins (MUC1, MUC2, MUC5AC, and MUC6) and type 1 Lewis antigens in cases

5. De Bolos C, Garrido M, Real FX: MUC6 apomucin shows a distinct normal

tissue distribution that correlates with Lewis antigen expression in the human stomach. Gastroenterology 1995, $109(3): 723-734$.

330

331

332

333

334

335

336

337

338

339

340

341

342

343

8. Virili C, Antonelli A, Santaguida MG, Benvenga S, Centanni M:

Gastrointestinal Malabsorption of Thyroxine. Endocr Rev 2019, 40(1):118136.

9. Robbe C, Capon C, Coddeville B, Michalski JC: Structural diversity and specific distribution of 0-glycans in normal human mucins along the intestinal tract. Biochem $J$ 2004, 384 (Pt 2):307-316.

10. Nakayama J: Dual Roles of Gastric Gland Mucin-specific 0-glycans in Prevention of Gastric Cancer. Acta Histochem Cytochem 2014, 47 (1):1-9.

11. Oue N, Sentani K, Sakamoto N, Uraoka N, Yasui W: Molecular carcinogenesis of gastric cancer: Lauren classification, mucin phenotype expression, and cancer stem cells. Int J Clin Oncol 2019, 24(7):771-778.

13. Andrianifahanana M, Moniaux N, Batra SK: Regulation of mucin expression: mechanistic aspects and implications for cancer and inflammatory diseases. Biochim Biophys Acta 2006, 1765(2):189-222.

14. Betge J, Schneider NI, Harbaum L, Pollheimer MJ, Lindtner RA, Kornprat P, Ebert MP, Langner C: MUC1, MUC2, MUC5AC, and MUC6 in colorectal cancer: expression profiles and clinical significance. Virchows Arch 2016, 469 (3) : 255-265.

15. Pinho SS, Reis CA: Glycosylation in cancer: mechanisms and clinical implications. Nat Rev Cancer 2015, 15 (9):540-555.

16. Ha Kim G, Am Song G, Youn Park D, Han Lee S, Hyun Lee D, Oh Kim T, Jae Jo H, Heo J, Hwan Kang D, Cho M: CDX2 expression is increased in gastric cancers with less invasiveness and intestinal mucin phenotype. Scandinavian journal of gastroenterology 2006, 41(8):880-886. 
344 17. Kim DH, Shin N, Kim GH, Song GA, Jeon TY, Kim DH, Lauwers GY, Park DY:

345

346

347

348

349

350

351

352

353

354

355

356

357

358

359

360

361

362

363

364

365

366

367

368

369

370

371

372

373

374

375

376

377

378

379

380

381

382

383

384

Mucin expression in gastric cancer: reappraisal of its clinicopathologic and prognostic significance. Arch Pathol Lab Med 2013, 137 (8):1047-1053.

18. Koseki K, Takizawa T, Koike M, Ito M, Nihei Z, Sugihara K: Distinction of differentiated type early gastric carcinoma with gastric type mucin expression. Cancer 2000, 89(4):724-732.

19. Yamazaki K, Tajima Y, Makino R, Nishino N, Aoki S, Kato M, Sakamoto M, Morohara K, Kaetsu T, Kusano M: Tumor differentiation phenotype in gastric differentiated-type tumors and its relation to tumor invasion and genetic alterations. World journal of gastroenterology 2006, $12(24): 3803-3809$.

20. Oue N, Sentani K, Sakamoto N, Yasui W: Clinicopathologic and molecular characteristics of gastric cancer showing gastric and intestinal mucin phenotype. Cancer Sci 2015, 106 (8):951-958.

21. Oue N, Sentani K, Sakamoto N, Uraoka N, Yasui W: Molecular carcinogenesis of gastric cancer: Lauren classification, mucin phenotype expression, and cancer stem cells. Int J Clin Oncol 2019, 24(7):771-778.

22. Hondo FY, Kishi H, Safatle-Ribeiro AV, Pessorrusso FCS, Ribeiro U, Maluf-Filho F: Characterization of the Mucin Phenotype Can Predict Gastric Cancer Recurrence after Endoscopic Mucosal Resection. Arq Gastroenterol 2017, $54(4): 308-314$.

23. Linden SK, Sutton P, Karlsson NG, Korolik V, McGuckin MA: Mucins in the mucosal barrier to infection. Mucosal Immunol 2008, 1(3):183-197.

24. Reis CA, David L, Correa P, Carneiro F, de Bolos C, Garcia E, Mandel U, Clausen H, Sobrinho-Simoes M: Intestinal metaplasia of human stomach displays distinct patterns of mucin (MUC1, MUC2, MUC5AC, and MUC6) expression. Cancer research 1999, 59(5):1003-1007.

25. Yamanoi K, Nakayama J: Reduced alphaGlcNAc glycosylation on gastric gland mucin is a biomarker of malignant potential for gastric cancer, Barrett's adenocarcinoma, and pancreatic cancer. Histochem Cell Biol 2018, 149 (6) :569-575.

26. Correa P, Houghton J: Carcinogenesis of Helicobacter pylori. Gastroenterology 2007, $133(2): 659-672$.

27. Correa P: Human gastric carcinogenesis: a multistep and multifactorial process--First American Cancer Society Award Lecture on Cancer Epidemiology and Prevention. Cancer research 1992, 52(24):6735-6740.

28. Chen XH, Wang A, Chu AN, Gong YH, Yuan Y: Mucosa-Associated Microbiota in Gastric Cancer Tissues Compared With Non-cancer Tissues. Front Microbiol 2019, 10:1261.

29. Filipe MI, Munoz N, Matko I, Kato I, Pompe-Kirn V, Jutersek A, Teuchmann $\mathrm{S}$, Benz M, Prijon T: Intestinal metaplasia types and the risk of gastric 
385

386

387

388

389

390

391

392

393

394

395

396

397

398

399

400

401

402

403

404

405

406

407

408

409

410

411

412

413

414

415

416

417

418

419

420

421

422

423

424

425

cancer: a cohort study in Slovenia. International journal of cancer 1994, 57 (3) :324-329.

30. Silva E, Teixeira A, David L, Carneiro F, Reis CA, Sobrinho-Simoes J, Serpa J, Veerman E, Bolscher J, Sobrinho-Simoes M: Mucins as key molecules for the classification of intestinal metaplasia of the stomach. Virchows Arch 2002, 440(3):311-317.

31. Gutierrez-Gonzalez L, Wright NA: Biology of intestinal metaplasia in 2008: more than a simple phenotypic alteration. Digestive and liver disease : official journal of the Italian Society of Gastroenterology and the Italian Association for the Study of the Liver 2008, 40(7):510522.

32. Altayar 0, Davitkov P, Shah SC, Gawron AJ, Morgan DR, Turner K, Mustafa RA: AGA Technical Review on Gastric Intestinal Metaplasia-Epidemiology and Risk Factors. Gastroenterology 2020, 158(3) :732-744 e716.

33. Gawron AJ, Shah SC, Altayar 0, Davitkov P, Morgan D, Turner K, Mustafa RA: AGA Technical Review on Gastric Intestinal Metaplasia-Natural History and Clinical Outcomes. Gastroenterology 2020, 158(3) :705-731 e705.

34. Banks M, Graham D, Jansen M, Gotoda T, Coda S, di Pietro M, Uedo N, Bhandari P, Pritchard DM, Kuipers EJ, Rodriguez-Justo M, Novelli MR, Ragunath K, Shepherd N, Dinis-Ribeiro M: British Society of Gastroenterology guidelines on the diagnosis and management of patients at risk of gastric adenocarcinoma. Gut 2019, 68(9) :1545-1575.

35. Dinis-Ribeiro M, Areia M, de Vries AC, Marcos-Pinto R, Monteiro-Soares M, O’ Connor A, Pereira C, Pimentel-Nunes P, Correia R, Ensari A, Dumonceau JM, Machado JC, Macedo G, Malfertheiner P, Matysiak-Budnik T, Megraud F, Miki K, O’ Morain C, Peek RM, Ponchon T, Ristimaki A, Rembacken B, Carneiro F, Kuipers EJ, European Society of Gastrointestinal E, European Helicobacter Study G, European Society of P, Sociedade Portuguesa de Endoscopia D: Management of precancerous conditions and lesions in the stomach (MAPS): guideline from the European Society of Gastrointestinal Endoscopy (ESGE), European Helicobacter Study Group (EHSG), European Society of Pathology (ESP), and the Sociedade Portuguesa de Endoscopia Digestiva (SPED). Endoscopy 2012, $44(1): 74-94$.

36. Pimente1-Nunes P, Libanio D, Marcos-Pinto R, Areia M, Leja M, Esposito G, Garrido M, Kikuste I, Megraud F, Matysiak-Budnik T, Annibale B, Dumonceau JM, Barros R, Flejou JF, Carneiro F, van Hooft JE, Kuipers EJ, Dinis-Ribeiro M: Management of epithelial precancerous conditions and lesions in the stomach (MAPS II): European Society of Gastrointestinal Endoscopy (ESGE), European Helicobacter and Microbiota Study Group

PeerJ reviewing PDF | (2020:07:51546:1:3:NEW 20 Dec 2020) 
426

427

428

429

430

431

432

433

434

435

436

437

438

439

440

441

442

443

444

445

446

447

448

449

450

451

452

453

454

455

456

457

458

459

460

461

462

463

464

465

466

467

(EHMSG), European Society of Pathology (ESP), and Sociedade Portuguesa de Endoscopia Digestiva (SPED) guideline update 2019. Endoscopy 2019, 51 (4) : 365-388. 
468 magnification; the images on the right were magnified images ( $₫ 400)$ of the boxed sections 469 depicted at left.

470

471

\section{Acid mucus phenotypes in the lesion adjacent to GC}

473 A,B. Acid mucus of type II can be seen in the lesion adjacent to GC. C,D. Acid mucus of type III can be seen in 474 the lesion adjacent to GC. Images on the left panel were made at $\mathbf{x} 100$ magnification; the images on 475 the right were magnified images $(\boldsymbol{x} 400)$ of the boxed sections depicted at left. 


\section{Table 1 (on next page)}

Table 1. Baseline characteristics of 483 subjects 
Table 1. Baseline characteristes of 483 subjects

\begin{tabular}{lccccc}
\hline Characteristes & SG & AG & GD & GC & $P$ value \\
\hline Total cases & 82 & 81 & 168 & 152 & \\
Age (years) & 52.09 & 61.46 & 61.73 & 62.39 & 0.000 \\
Sex (\%) & & & & & \\
Male & $42(51.22)$ & $46(56.79)$ & $112(66.67)$ & $99(65.13)$ & 0.067 \\
Female & $40(48.78)$ & $35(43.21)$ & $56(33.33)$ & $53(34.87)$ & \\
\hline
\end{tabular}


Table 2 (on next page)

Table 2. Mucous phenotype characteristics of different gastric diseases 
Table 2. Mucous phenotype characteristics of different gastric diseases

\begin{tabular}{cccccc}
\hline Diseases & Cases & neutral mucus $(\%)$ & acidic mucus $(\%)$ & non-mucus $(\%)$ & P value \\
\hline Total & 483 & $135(27.89)$ & $154(31.82)$ & $195(40.29)$ & \\
SG & 82 & $82(100)$ & 0 & 0 & 0.000 \\
AG & 81 & $12(14.81)$ & $66(81.48)$ & $3(3.71)$ & 0.000 \\
GD & 168 & $36(21.43)$ & $59(35.12)$ & $73(43.45)$ & 0.000 \\
GC & 152 & $4(2.63)$ & $29(19.08)$ & $119(78.29)$ & 0.000 \\
$P$ value & & 0.000 & 0.000 & 0.000 & \\
\hline
\end{tabular}




\section{Table 3 (on next page)}

Table 3. The analysis of mucous phenotype characteristics of different gastric diseases 
Table 3. Subgroup analysis of mucous phenotype characteristics of different gastric diseases

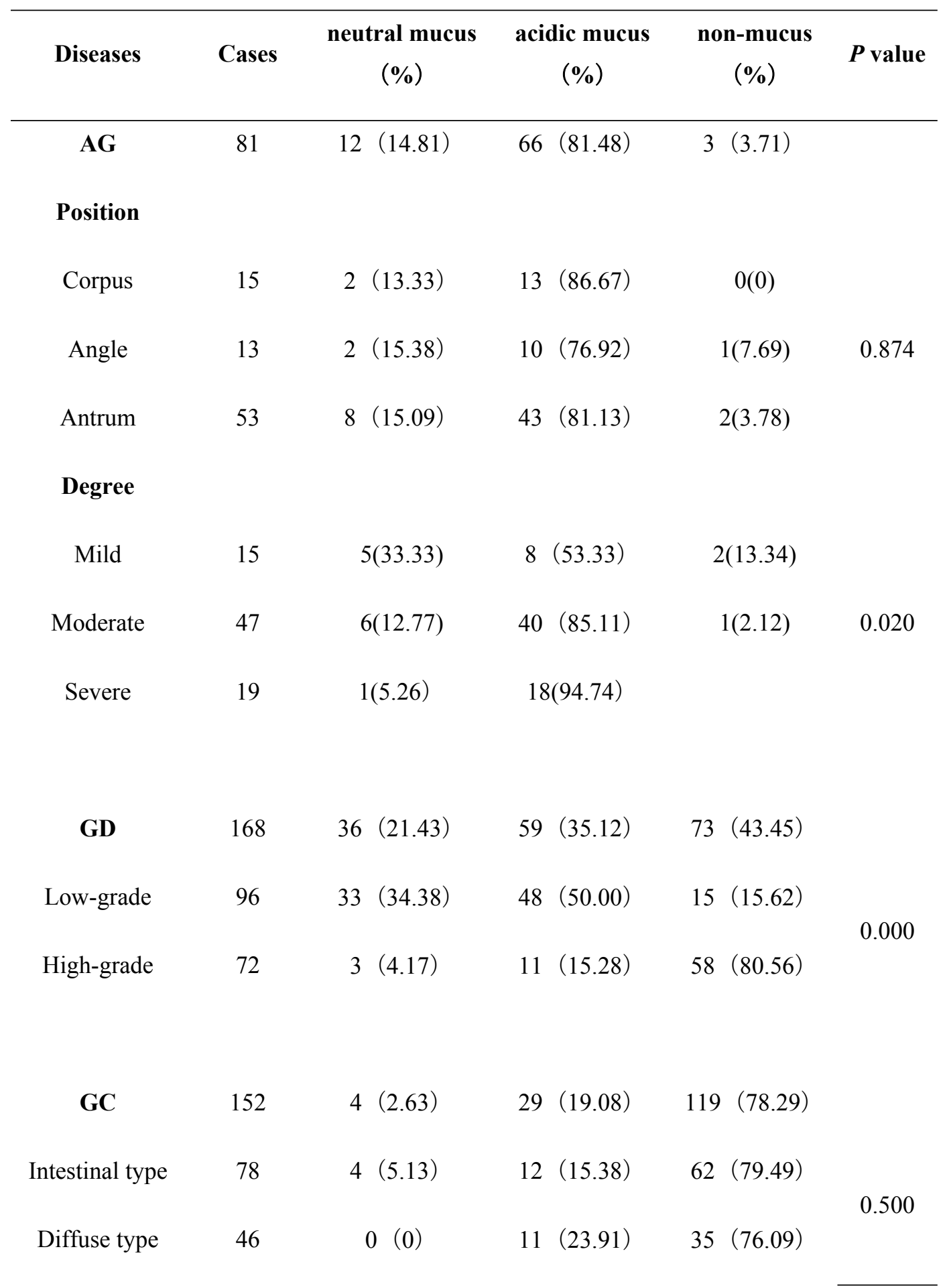




$\begin{array}{cccccc}\text { Mixed type } & 3 & 0 & (0) & 1(33.33) & 2(66.67) \\ \text { Unclassifiable } & 25 & 0 & (0) & 5(20.00) & 20\end{array}$

2 


\section{Table 4 (on next page)}

Table 4. Subgroup analysis of mucous phenotype characteristics of different H.pylori status 
Table 4. Subgroup analysis of mucous phenotype characteristics of different Hp status

\begin{tabular}{|c|c|c|c|c|c|c|}
\hline Diseases & Нр & Cases & $\begin{array}{c}\text { neutral mucus } \\
(\%)\end{array}$ & acidic mucus $(\%)$ & mucus-free (\%) & $P$ value \\
\hline \multirow{3}{*}{ Total } & Positive & 97 & $15(15.46)$ & $41(42.27)$ & $41(42.27)$ & \multirow{3}{*}{0.724} \\
\hline & & & & & & \\
\hline & Negative & 53 & $7(13.21)$ & $20(37.73)$ & $26(49.06)$ & \\
\hline \multirow{3}{*}{$\mathbf{A G}$} & Positive & 37 & $8(21.62)$ & $27(72.97)$ & $2(5.41)$ & \multirow{3}{*}{0.000} \\
\hline & & & & & & \\
\hline & Negative & 8 & $8(100)$ & $0(0)$ & $0(0)$ & \\
\hline \multirow{3}{*}{ GD } & Positive & 33 & $6(18.18)$ & $10(30.30)$ & $17(51.52)$ & \multirow{3}{*}{0.879} \\
\hline & & & & & & \\
\hline & Negative & 26 & $6(23.08)$ & $8(30.77)$ & $12(46.15)$ & \\
\hline \multirow{3}{*}{ GC } & Positive & 27 & $1(3.71)$ & $4(14.81)$ & $22(81.48)$ & \multirow{3}{*}{0.819} \\
\hline & & & & & & \\
\hline & Negative & 19 & $1(5.26)$ & $4(21.05)$ & $14(73.69)$ & \\
\hline
\end{tabular}




\section{Table 5 (on next page)}

Table 5. The distribution of acidic mucus phenotype in different diseases 
Table 5. The distribution of acidic mucus phenotype in different diseases

\begin{tabular}{|c|c|c|c|c|c|}
\hline Diseases & Cases & Type I（\%） & Type II （\%) & Type III（\%) & $P$ value \\
\hline CAG & 66 & $11(16.67)$ & $10(15.15)$ & $45 \quad(68.18)$ & 0.000 \\
\hline \multicolumn{6}{|l|}{ Position } \\
\hline Corpus & 13 & $0 \quad(0)$ & $2(15.38)$ & $11(84.62)$ & \\
\hline Angle & 10 & $1(10)$ & $2(20)$ & $7(70)$ & 0.348 \\
\hline Antrum & 43 & $10(23.26)$ & $6(13.95)$ & $27(62.79)$ & \\
\hline \multicolumn{6}{|l|}{ Degree } \\
\hline Mild & 8 & $3(37.5)$ & $1(12.5)$ & $4(50)$ & \\
\hline Moderate & 40 & $5(12.5)$ & $7(17.5)$ & $28(70)$ & 0.505 \\
\hline Severe & 18 & $3(16.67)$ & $2(11.11)$ & $13(72.22)$ & \\
\hline GD & 59 & $3(5.08)$ & $12(20.34)$ & $44 \quad(74.58)$ & 0.000 \\
\hline Low-grade & 48 & $3(6.25)$ & $12(25)$ & $33 \quad(68.75)$ & \\
\hline & & & & & 0.014 \\
\hline High-grade & 11 & $0 \quad(0)$ & $0 \quad(0)$ & $11(100)$ & \\
\hline GC & 29 & $2(6.90)$ & $6(20.69)$ & $21(72.41)$ & 0.000 \\
\hline Intestinal type & 12 & $0(0)$ & $3(25)$ & $9(75)$ & \\
\hline Diffuse type & 11 & $0(0)$ & $2(18.18)$ & $9(81.82)$ & \\
\hline & & & & & 0.091 \\
\hline Mixed type & 1 & $0 \quad(100)$ & $0 \quad(0)$ & $1(100)$ & \\
\hline Unclassifiable & 5 & $2(40)$ & $1(20)$ & $2(40)$ & \\
\hline
\end{tabular}




\section{Table 6(on next page)}

Table 6. The comparation of acid mucus phenotypes in the lesion adjacent to high-grade GD or GC 
Table 6. The comparation of acid mucus phenotypes in the lesion next to high-grade GD or GC

\begin{tabular}{|c|c|c|c|c|c|}
\hline GD/GC & Cases & Type I（\%) & Type II （\%) & Type III（\%) & $P$ value \\
\hline High-grade & 42 & $4(9.53)$ & $3(7.14)$ & $35 \quad(83.33)$ & 0.000 \\
\hline Intestinal type & 49 & $4(8.16)$ & $1 \quad(2.04)$ & $44 \quad(89.8)$ & \\
\hline \multirow[t]{2}{*}{ Diffuse type } & 13 & $1 \quad(7.69)$ & $0 \quad(0)$ & $12(92.31)$ & \\
\hline & & & & & 0.980 \\
\hline Mixed type & 2 & $0(0)$ & 0 & $2(100)$ & \\
\hline Unclassifiable & 6 & $1 \quad(16.67)$ & 0 & $5(83.33)$ & \\
\hline
\end{tabular}

2 


\section{Figure 1}

1. Mucous phenotype characteristics of different gastric diseases

A, B. Representative picture of neutral mucus (red) in the SG group. C, D. Representative picture of acid mucus (blue) in the AG group. E, F. Representative picture of mucus-free in GC group. Images on the left panel were made at ${ }_{\star} 100$ magnification; the images on the right were magnified images $(\times 400)$ of the boxed sections depicted at left. 

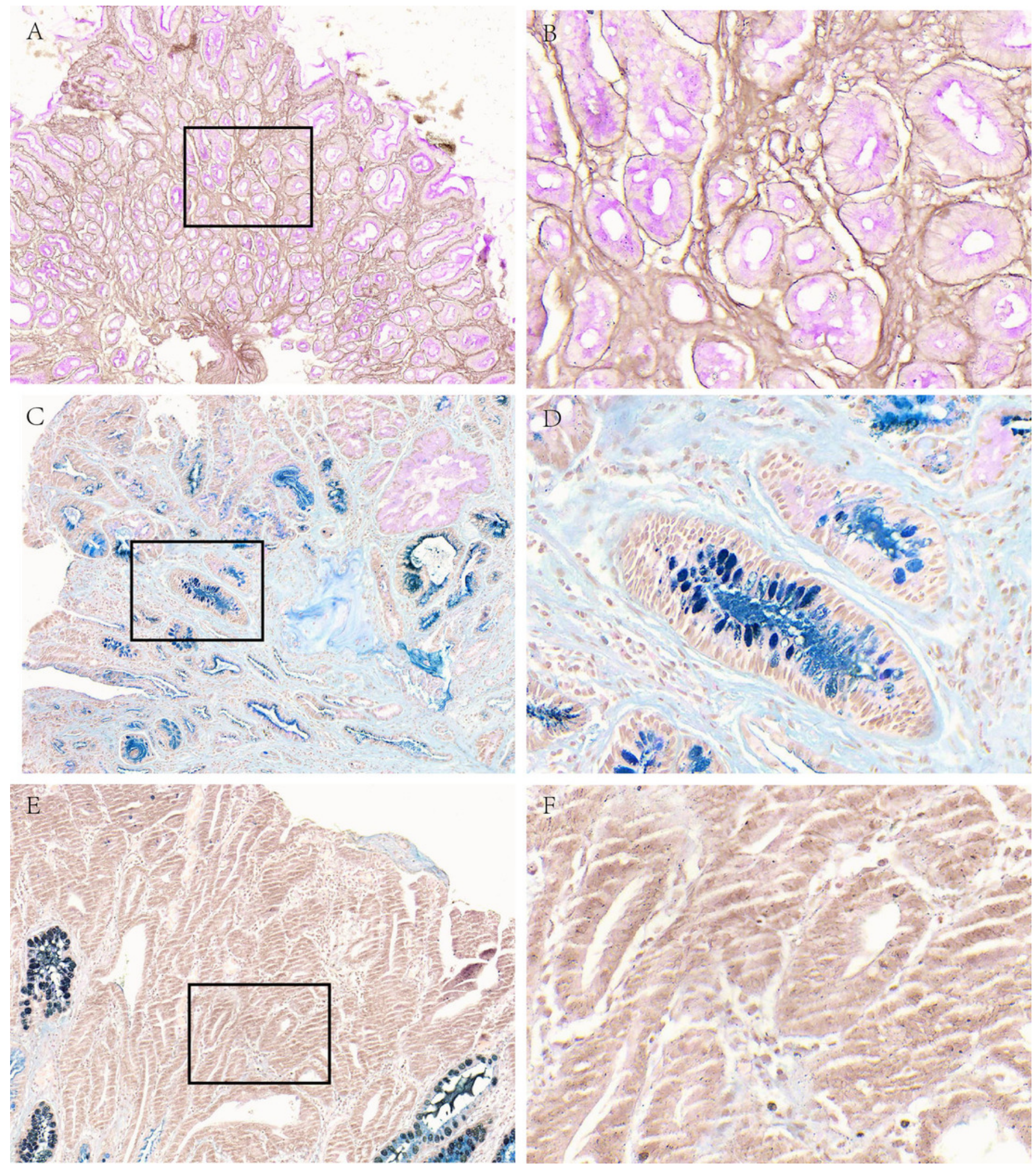
Figure 2

2. Acidic mucus phenotype in AG

A, B. Representative picture of type I (blue). C, D. Representative picture of type II (redblue).E, F. Representative picture of type III(red-brown black). Images on the left panel were made at ${ }_{x} 100$ magnification; the images on the right were magnified images $\left({ }_{x} 400\right)$ of the boxed sections depicted at left. 

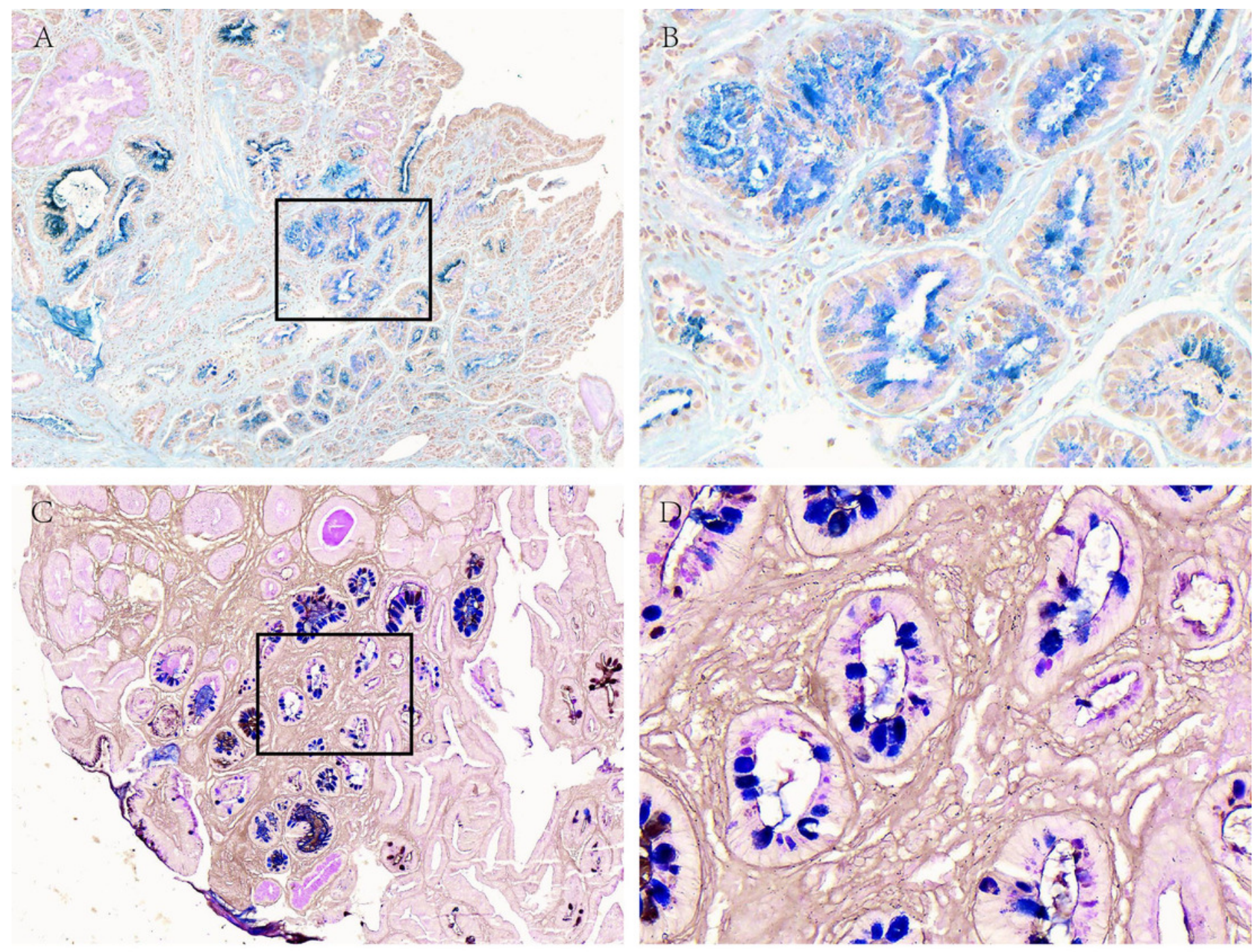

E
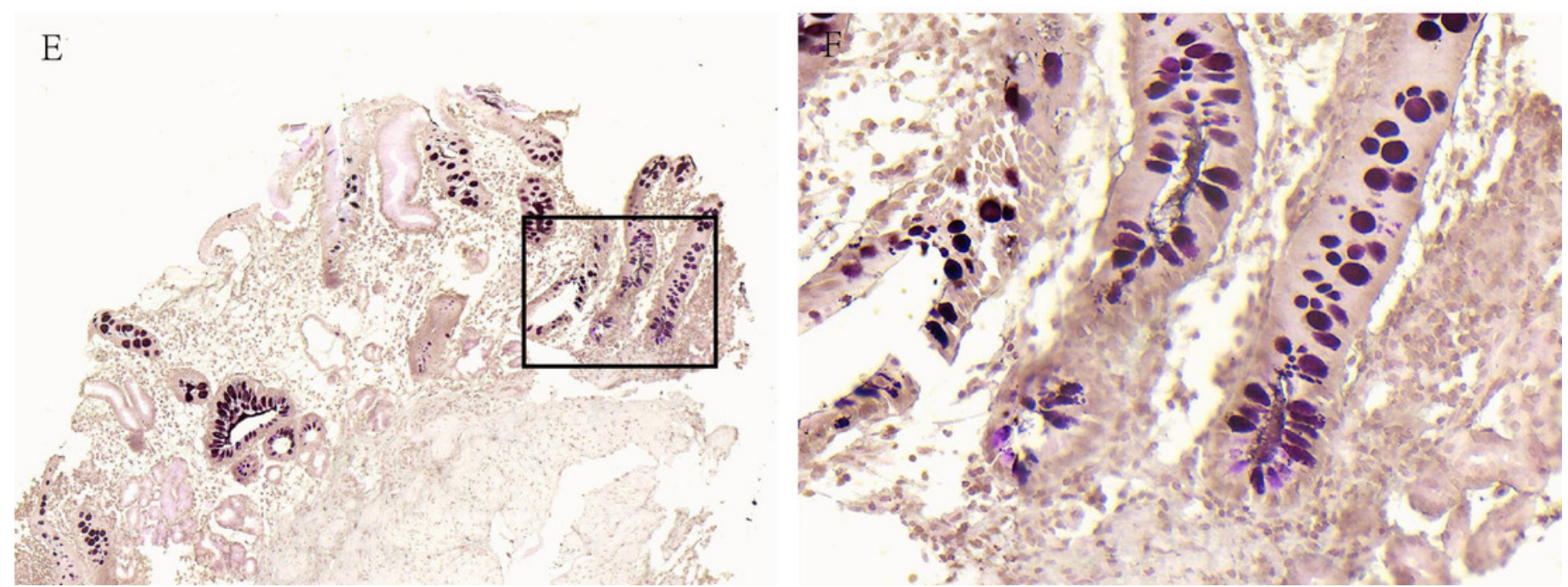


\section{Figure 3}

3. Acid mucus phenotypes in the lesion adjacent to GC

A,B. Acid mucus of type II can be seen in the lesion adjacent to GC. C,D. Acid mucus of type III can be seen in the lesion adjacent to GC.Images on the left panel were made at ${ }_{x} 100$ magnification; the images on the right were magnified images $\left({ }_{x} 400\right)$ of the boxed sections depicted at left.
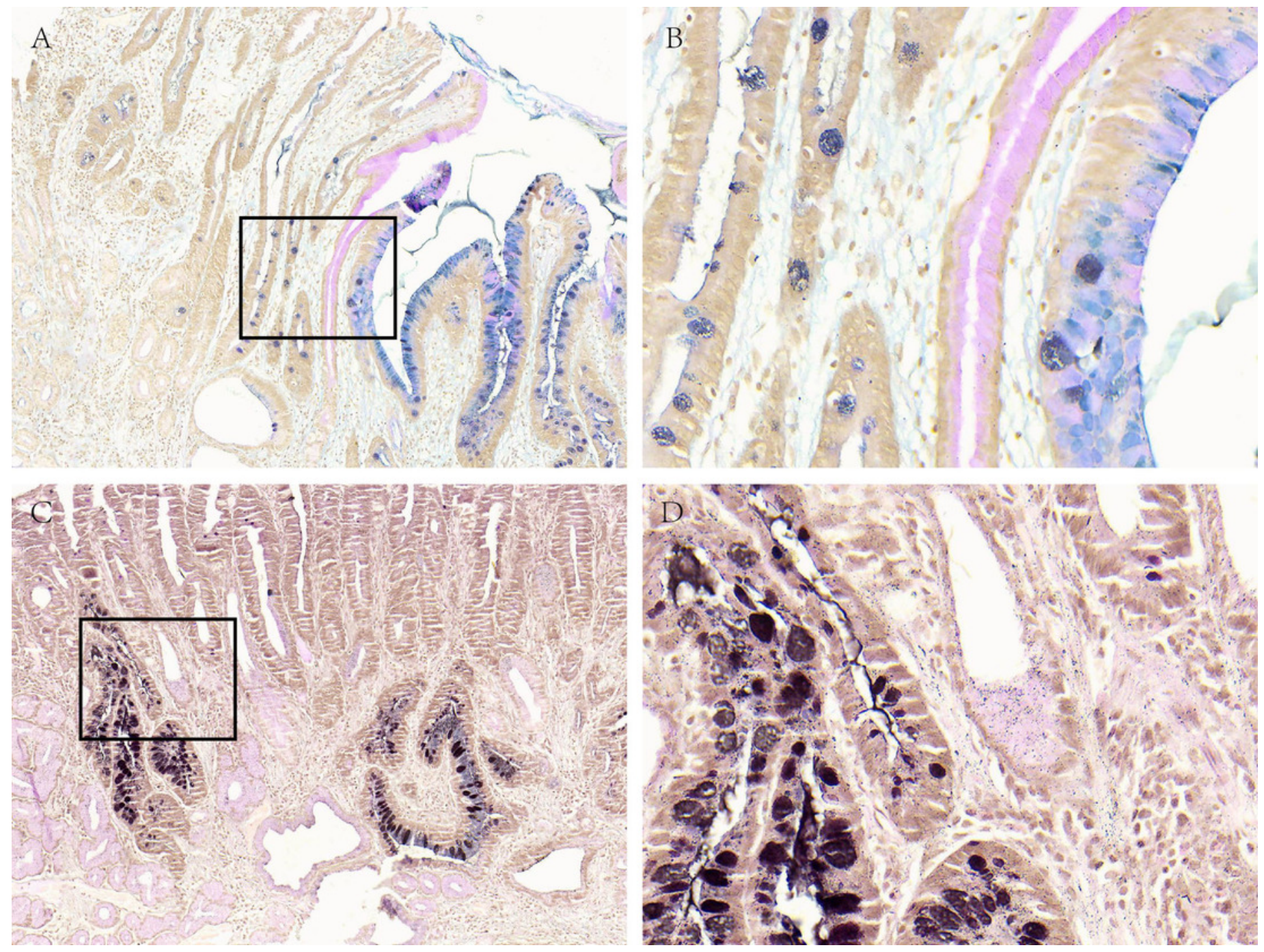